\title{
La Grammatica in Dante
}

\section{Raffaele Pinto}

Universitat de Barcelona

pinto.raffaele@gmail.com

\begin{abstract}
La Grammatica occupa in Dante uno dei due poli del paradigma diglossico caratteristico della cultura linguistica del Medioevo. La riflessione sulla Grammatica si sviluppa creativamente dalla Vita Nuova alla Commedia, a partire da concetti aristotelico-tomisti e in direzione della definitiva neutralizzazione delle diglossie messe in evidenza soprattutto nel Convivio e nel De vulgari. Tale neutralizzazione verrà teorizzata nel canto XXVI del Paradiso.
\end{abstract}

Parole chiave: grammatica; volgare; diglossia; Babele; Adamo.

\section{Abstract}

Grammar in Dante occupies one of the two poles of the diglossic paradigm characteristic of the linguistic culture of the Middle Ages. Reflection on grammar is developed creatively from the Vita Nuova to the Commedia, starting from Aristotelian-Thomist concepts and leading to the definitive neutralisation of the diglossia demonstrated especially in the Convivio and the De vulgari. This neutralisation will be theorised in Canto XXVI of the Paradiso.

Keywords: grammar; vulgar; diglossia; Babel; Adam. 


\section{Il dualismo Grammatica - Volgare nella Vita Nuova}

Il concetto di grammatica raramente appare in Dante isolato e definito in se stesso come una delle dottrine in cui si articolava il sapere della sua epoca. Ciò accade in Convivio II XIII 9, dove la grammatica viene comparata al cielo della luna e descritta sviluppando concetti oraziani:

Dico che 'l cielo della Luna con la Gramatica si somiglia, perché ad esso si può comparare [per due proprietadi]. Che se la Luna si guarda bene, due cose si veggiono in essa proprie, che non si veggiono nell'altre stelle. L'una si è l'ombra che è in essa, la quale non è altro che raritade del suo corpo, a la quale non possono terminare li raggi del sole e ripercuotersi così come ne l'altre parti; l'altra si è la variazione della sua luminositade, che ora luce da uno lato e ora luce da un altro, secondo che lo sole la vede. E queste due proprietadi hae la Gramatica: ché per la sua infinitade li raggi de la ragione in essa non si terminano, in parte spezialmente de li vocabuli; e luce or di qua or di là, in tanto [in] quanto certi vocabuli, certe declinazioni, certe construzioni sono in uso che già non furono, e molte già furono che ancor saranno: sì come dice Orazio nel principio de la Poetria, quando dice: «Molti vocabuli rinasceranno che già caddero».

Normalmente la grammatica è invece uno dei poli di un sistema linguistico e culturale che ha, ai suoi estremi, il latino e il volgare, ed indica appunto il latino inteso come lingua scolastica e letteraria che si oppone al volgare inteso come lingua spontanea e quotidiana. Conviene partire dal primo testo in cui tale dicotomia è formulata, che illustra bene il paradigma diglossico all'interno del quale il pensiero di Dante sulla Grammatica matura ed evolve. ${ }^{2}$ Si tratta di un passaggio del capitolo XXV della Vita Nuova in cui il poeta si interroga sulle origini della poesia d'amore scritta in volgare:

Anticamente non erano dicitori d'amore in lingua volgare, anzi erano dicitori d'amore certi poete in lingua latina; tra noi dico, avvegna forse che tra altra gente addivenisse, e addivegna ancora, sì come in Grecia, non volgari ma litterati poete queste cose trattavano. ${ }^{3}$

1. Cito da Dante Alighieri, Opere Minori, Tomo I - Parte II, a cura di Cesare Vasoli e di Domenico De Robertis, Milano-Napoli: Ricciardi, 1988. Il brano interessa soprattutto per la teoria cosmologica di Dante, che qui, relativamente alle macchie lunari, adduce argomenti di tipo fisico-quantitativo mentre in Par. II addurrà principi di ordine metafisico-qualitativo. Per quanto riguarda la teoria grammaticale, contrariamente alla linea principale della sua ricerca, nella quale la grammatica si identifica con il latino lingua artificiale e inalterabile, qui «si postula una fluttuazione nell'uso, che riguarda non solo il vocabolario ma anche la sintassi e la morfologia, e che la grammatica riflette» (Pier Vincenzo Mengaldo, v. Lingua. Le teorie dantesche sulla lingua, in Enciclopedia Dantesca, Roma: Istituto della Enciclopedia italiana, 1965).

2. Sulla diglossia strutturale della cultura medievale si veda Gian Carlo Alessio, Tradizione latina e origini romanze, in Franco Brioschi e Costanzo Di Girolamo (a cura di), Manuale di letteratura italiana. Storia per generi e problemi, I, Torino: Bollati-Boringhieri, 1993, p. 3-44.

3. Sulla sintassi di questo passaggio della Vita Nuova, e sul principio diglossico ad esso inerente, si veda Mirko Tavoni, "Vita Nuova’ XXV 3 e altri appunti di linguistica dantesca», Rivista di letteratura italiana, II, n. 1, 1984, p. 9-52. 
Il testo allude alla originalità storica della poesia d'amore in volgare, dal momento che, come dirà subito dopo, sono passati appena 150 anni da quando fu presa l'iniziativa di usare letterariamente il volgare. E si tratta di una originalità non solo storica, cioè databile, ma anche geografica, dal momento che in altre zone d'Europa i poeti continuano ad usare una lingua grammaticale e non volgare. È il caso della Grecia, nella quale secondo Dante tale mutamento di lingua letteraria non si è verificato.

Il brano è estremamente interessante poiché ci mostra, sul nascere, il paradigma diglossico della idea dantesca di lingua e linguaggio: l'Europa ha un numero indeterminato di lingue locali (naturali), che nel loro insieme possono essere definite come il «volgare» e due sole grammatiche, cioè il latino e il greco, che sono le lingue dei litterati. La letteratura si è tradizionalmente scritta in tali due lingue grammaticali, che ad essa, anzi, sono strutturalmente destinate, finché "tra noi», ossia nell'ambito geografico e culturale che adotta come lingua grammaticale il latino, i poeti hanno abbandonato la grammatica (linguaggio d'arte, cioè artificialmente costruito) per il volgare (linguaggio d'uso naturale). L'idea che ad una certa lingua grammaticale appartengano diversi idiomi locali è ben attestata prima di Dante (per esempio in Ruggero Bacone) $;{ }^{4}$ ma che una di queste grammatiche sia stata soppiantata, per certi usi letterari, dal volgare, che entra così in concorrenza con la grammatica sul terreno della letteratura, questa è prospettiva dantesca e che poteva essere sviluppata solo a partire dalla esperienza lirica (che legittima, come vedremo subito, l'uso preferenziale del volgare in ragione del tema trattato e del destinatario del testo).

Del fatto che esista una lingua grammaticale «nostra», che si oppone alle grammatiche altrui, e concretamente in Europa al greco, si parla anche in un altro discusso luogo del Convivio, che risulta più chiaro alla luce del passaggio appena letto della Vita Nuova. In Conv. I xI 14, in polemica con i detrattori dell'italiano che preferiscono il provenzale, che è in Italia «volgare altrui», Dante cita il De Finibus di Cicerone, in cui viene denunciata l'insolenza di quanti, pur essendo latini, preferiscono il greco al latino, in testi di tipo filosofico: ${ }^{5}$

4. «In lingua enim Latina quae una est, sunt multa idiomata. Substancia enim ipsius lingue consistit in hiis in quibus communicant clerici et litterati omnes. Idiomata vero sunt multa secundum multitudinem nacionum utencium hac lingua. Quia aliter in multis pronunciant et scribunt ytalici, et aliter gallici, et aliter teutonici, et eliter anglici, et ceteri» [Nella lingua latina, che è una sola, esistono molti idiomi. La sostanza di questa lingua consiste in quello che i chierici e tutti i letterati utilizzano per comunicare. Gli idiomi, in verità, sono molti a seconda delle diverse nazioni che utilizzano questa lingua, perché in un modo la pronunciano e scrivono gli italiani, in un altro gli spagnoli, in un altro i francesi, in un altro $\mathrm{i}$ tedeschi, in un altro ancora gli inglesi] (Summa gramatica; il brano è citato e commentato, con altri della stessa epoca, in Giancarlo Alessio, op.cit., p. 18-25).

5. I 4: «Iis igitur est difficilius satis facere, qui se Latina scripta dicunt contemnere. In quibus hoc primum est in quo admirer, cur in gravissimis rebus non delectet eos sermo patrius, cum idem fabellas Latinas ad verbum e Graecis expressas non inviti legant. Quis enim tam inimicus paene nomini Romano est, qui Ennii Medeam aut Antiopam Pacuvii spernat aut reiciat, quod se isdem Euripidis fabulis delectari dicat, Latinas litteras oderit?». 
Contra questi cotali grida Tullio nel principio d'un suo libro che si chiama Libro di Fine de' Beni, però che al suo tempo biasimavano lo latino romano e commendavano la gramatica greca, per simiglianti cagioni che questi fanno vile lo parlare italico e prezioso quello di Provenza.

Come ha ben avvertito Pier Vincenzo Mengaldo, il confronto è fra due grammatiche (una nostra, il latino, una altrui, il greco), paragonate in blocco a due volgari (uno nostro, l'italiano, uno altrui, il provenzale). ${ }^{6}$ Ciò che è in discussione, in entrambi i casi, è la naturale e quindi necessaria difesa di ciò che è proprio contro ciò che è altrui.

Essendo dunque latino e greco le lingue grammaticali d'Europa, in occidente è accaduto che i poeti d'amore hanno abbandonato la lingua grammaticale e l'hanno sostituita con il volgare. Tale visione "catastrofica» della poesia moderna ha come presupposto teorico la diglossia strutturale delle società umane, nelle quali sempre e dovunque si oppone una lingua scritta e letteraria ad un'altra parlata e di uso quotidiano. L'originalità culturale dell'occidente europeo consiste, secondo Dante, nell'uso letterario della lingua quotidiana, e quindi nel corto circuito che si stabilisce tra funzioni di linguaggio che tradizionalmente (cioè anticamente e, nella attualità, in ambito non latino) sono finalizzate ad usi espressivi radicalmente diversi, e cioè l'artificialità del latino per la scrittura, e la spontaneità del parlato per la comunicazione quotidiana.

$\grave{E}$ in tale prospettiva che acquista il massimo rilievo quella che, nelle linee immediatamente successive della Vita Nuova, viene indicata come la causa scatenante di tale rottura della continuità culturale di occidente, sul piano linguistico-letterario:

Lo primo che cominciò a dire siccome poeta volgare, si mosse però che volle fare intendere le sue parole a donna, a la quale era malagevole d'intendere li versi latini. E questo è contra coloro che rimano sopra altra matera che amorosa, con ciò sia cosa che cotale modo di parlare fosse dal principio trovato per dire d'amore.

Le ragioni del mutato rapporto fra latino e volgare, e cioè le cause della invasione da parte del volgare di un territorio tradizionalmente e strutturalmente riservato al latino, consistono per Dante nella selezione di un nuovo destinatario poetico, che, non conoscendo il latino, non avrebbe potuto intendere i testi. Il primo poeta volgare cambiò lingua non perché non conoscesse il latino, ma perché si dirigeva ad un interlocutore che lo ignorava, ossia una donna. Nella prospettiva di Dante, il latino era lingua specificamente maschile, poco frequentata dalle donne, e per tanto, a chi volesse parlare letterariamente ad una donna e farsi capire da lei, era necessario usare l'unica lingua alla loro portata, ossia il volgare. Per capire questo ragionamento dobbiamo pensare che nella diversificazione diglossica della società europea, il latino era da secoli lingua specializzata dei chierici, insegnato in scuole quasi sempre

6. Cfr. Pier Vincenzo Mengaldo, op. cit. 
ecclesiastiche alle quali le donne non avevano accesso (almeno ai livelli più elevati). Per Dante, tale esclusione delle donne dalla letteratura, e quindi dalla grammatica, era elemento strutturale della società europea (e umana in generale), ed avverte quindi come una innovazione "catastrofica» l'irruzione delle donne nella cultura letteraria, in qualità di lettrici o destinatarie del discorso poetico.

Indipendentemente dalla oggettiva validità socioculturale di queste osservazioni, è indubbio che con esse viene inaugurata una linea di riflessione che si protrarrà per alcuni secoli nel dibattito letterario degli intellettuali europei. Ricordo solo alcuni di questi momenti perché sia chiara la assoluta pertinenza teorica dell'argomento relativo al destinatario femminile quale causa della svolta culturale realizzata dai trovatori. ${ }^{7}$ Innanzitutto Boccaccio, che in diversi luoghi dichiara che le donne sono destinatario prioritario dei romanzi, ${ }^{8} \mathrm{e}$ nel Proemio del Decameron seleziona le «donne innamorate» come pubblico dell'opera, la quale, d'altra parte, essendo "cognominata Principe Galeotto», allude fin dal titolo al privilegiato destinatario femminile dei suoi temi romanzeschi. Poi Baldesar Castiglione, che nel III libro del Cortegiano si chiede retoricamente:

chi studia a compor versi, almen nella lingua volgare, se non per esprimere quegli affetti che dalle donne sono causati?

e infine Joan Boscà, che nel Prólogo al secondo libro delle sue poesie, ispirato da Dante, Boccaccio e Castiglione, dà per scontato che la lirica abbia come destinatario prioritario le donne, e ne difende, contro i detrattori, la dignità intellettuale:

otros argüían diziendo que esto principalmente havía de ser para mugeres y que ellas no curavan de cosas de sustancia sino del son de las palanbras y de la dulçura del consonante... a los que dizen que estas cosas, no siendo sino para mugeres, no han de ser muy fundadas, ¿quién ha de gastar tiempo en respondelles? Tengo yo a las mugeres por tan sustanciales, las que aciertan a sello, y aciertan muchas, que en este caso quien se pusiese a defendellas las ofenderia.

7. L'argomento viene quasi sempre snobbato dai critici di Dante. Piace perciò, qui, ricordare le acute osservazioni di D. De Robertis al riguardo: «la tesi, che trova sostanziale conferma nel De vulg. el., I, I, 3, è "tutt'altro che risibile" (come parve ai più), e ritorna, dilatata nel senso di una "maggiore democraticità" (una democraticità, beninteso, tutta dantesca ... di "principi, baroni, cavalieri ... non solamente maschi ma femmine ... volgari e non letterati"), nel Convivio, I, XI, 4-5. Le donne cioè (le donne "che hanno intelletto d'amore" alla stagione della Vita Nuova) sono l'avanguardia, ormai simbolica, di questo nuovo schieramento".

8. Di particolare rilievo, perché l'ottica è misogina, sono queste osservazioni del Corbaccio sull'immaginario romanzesco-sessuale delle donne: «Ella [cioè "quella prodezza che le piace"] s'usa nelle camere, ne' nascosi luoghi, ne' letti e negli altri simili luoghi acconci a ciò, dove, senza corso di cavallo o suon di tromba di rame, alle giostre si va a pian passo; e colui tiene ella che sia o vuoi Lancelotto, o vuogli Tristano, o Orlando o Ulivieri, di prodezza, la cui lancia per sei o otto aringhi o per dieci in una notte non si piega in guisa che poi non si drizzi». 
L'irruzione del pubblico femminile nell'orizzonte comunicativo del poeta europeo viene percepita dalle «avanguardie» letterarie una svolta epocale che ha, come immediata e necessaria conseguenza, l'adozione letteraria della lingua delle donne, ossia il volgare inteso quale lingua materna. Tale rapporto originario e strutturale fra la letteratura in volgare ed il pubblico femminile costituisce uno dei pilastri della riflessione linguistica di Dante. Riapparirà infatti nel $1^{\circ}$ trattato del Convivio (I Ix 5), laddove viene selezionato il pubblico della nuova letteratura, una società civile al suo interno ampiamente differenziata, formata da

... molta altra nobile gente, non solamente maschi ma femmine, che sono molti e molte in questa lingua, volgari, e non letterati.

Esso viene ribadito all'inizio del De Vulgari (I I) quando si afferma che anche alle donne e ai bambini è necessaria una eloquenza volgare:

Talem eloquentiam penitus omnibus necessariam videamus, cum ad eam non tantum viri, sed etiam mulieres et parvuli nitantur, in quantum natura permictit. ${ }^{\text {? }}$

[Tale eloquenza è profondamente necessaria a tutti, dato che ad essa tendono non solo gli uomini ma anche, per quanto consente loro la natura, le donne e i bambini].

si precisa, poi, attraverso la nozione di lingua materna, nella definizione di volgare, nello stesso capitolo del $1^{\circ}$ libro del trattato latino:

Vulgarem locutionem asserimus, quam sine omni regula nutricem imitantes accipimus.

[Definiamo parlar volgare quello che assorbiamo al di fuori di qualunque regola, imitando la nutrice],

nella quale l'istanza naturale e femminile del linguaggio si oppone a quella artificiale e grammaticale. ${ }^{10} \mathrm{E}$ viene poi definitivamente legittimato, sul piano mitico e poetico, quindi ben al di là della astratta dimensione teorica, attraverso il personaggio di Francesca da Rimini nel canto V dell' Inferno, il primo di cui viene esplorato il carattere nella Commedia, lettrice di romanzi e teorica dell'amore.

Tale irruzione del pubblico femminile nella cultura letteraria, d'altra parte, è comprensibile solo nel quadro di una poesia che abbia l'amore come suo tema centrale (addirittura l'unico plausibile in volgare, per Dante, all'altezza della Vita Nuova). Il volgare viene promosso alla dignità di lingua letteraria, spodestando, almeno in quest'ambito, il latino, in quanto esprime l'esperienza del desiderio nei confronti di una donna, che è, da una parte, oggetto d'amore e dall'altra destinataria del testo poetico.

9. Cito da Mirko Tavoni (a cura di), De Vulgari Eloquentia, in Dante, Opere, I, Milano: Mondadori, 2011.

10. Ė questo il percorso concettuale suggerito da D. De Robertis (cfr. nota n. 7). 
Nella sua schematicità, quello formulato nel capitolo XXV della Vita Nuova è un modello ermeneutico densamente esplicativo sia sul piano storicoculturale che su quello linguistico-letterario: la antica diglossia, basata sulla opposizione chierici-laici, viene messa in discussione e superata dalla poesia dei trovatori e dalla tradizione che ad essa si ispira. Grazie ad esse viene prefigurato un nuovo sistema che, appellando a valori radicalmente alternativi (cioè l'amore inteso come fondamento di una nuova aristocrazia dei sentimenti e quindi di una nuova antropologia) e a nuovi soggetti culturali (cioè i laici in generale $^{11}$ e le donne in particolare), relega il latino in un ruolo culturalmente non più egemone. Esso servirà semmai, e resterà vigente, quale modello a cui ispirarsi nella elaborazione del nuovo linguaggio letterario (come gli esempi sulla figura della personificazione mostrano in questo stesso capitolo della Vita Nuova), ma non potrà più esprimere i valori essenziali e fondativi della civiltà, che attraverso la soggettività femminile (la donna come destinataria-lettrice, oltre che come oggetto di desiderio) si legano ormai organicamente ed indissolubilmente alla promozione e al progresso della lingua volgare. Tale sentimento della inattualità del latino doveva essere, oltre che convinzione personale di Dante, anche tema di dibattito fra i poeti di Firenze, se sempre nelle Vita Nuova leggiamo, nel cap. XXX, all'interno di una autogiustificazione per il fatto di non riportare per intero il brano delle Lamentationes di Geremia con cui lo scrittore ha informato il lettore della morte di Beatrice, la seguente dichiarazione:

Lo intendimento mio non fue dal principio di scrivere altro che per volgare; onde, con ciò sia cosa che le parole che seguitano a quelle allegate, siano tutte latine, sarebbe fuori dal mio intendimento se le scrivessi. E simile intenzione so ch'ebbe questo mio primo amico a cui io ciò scrivo, cioè ch'io li scrivessi solamente in volgare.

Questa sintonia fra Dante e Guido Cavalcanti sulla inopportunità dell'uso del latino sembrerebbe alludere ad una convinzione relativamente diffusa fra i cultori della lirica in volgare, e cioè al fatto che i temi e le esperienze di cui tale lirica è veicolo implicano un destinatario femminile e laico, e quindi uno strumento espressivo ad essi adeguato. ${ }^{12}$

11. È importante tener conto del mutamento di orizzonte sociologico di Dante, a partire dall'esilio (è questo il tema su cui ha insistito con grande acutezza e dovizia di argomenti Umberto Carpi, La nobiltà di Dante, Firenze: Polistampa, 2004): se a Firenze i suoi interlocutori sono sostanzialmente i borghesi del Comune, dopo il 1302 il suo discorso si rivolge soprattutto alla classe nobiliare italiana, nel quadro della svolta tendenzialmente ghibellina del suo pensiero. Osservo che si tratta, in ogni caso, di un interlocutore laico, essendo la polemica anticlericale una constante della sua riflessione sulla cultura letteraria e, con ogni evidenza, l'aspetto più modernamente innovatore del suo pensiero politico.

12. Sul destinatario femminile come elemento strutturale della lirica in volgare, in Italia, fin da Giacomo da Lentini, rinvio a Raffaele Pinto, Giacomo da Lentini. La parola del cuore, in Poetiche del desiderio, Roma: Aracne, 2010, p. 99-130. 
Nel pensiero di Dante il latino e la grammatica occupano il lato socialmente negativo della polarità diglossica; essi sono storicamente obsoleti e destinati ad essere soppiantati attraverso l'istituzione di un nuovo diasistema, non più diglossico, basato sulla identità, almeno tendenziale, della lingua d'uso o materna e la lingua d'arte o letteraria. La negatività della grammatica, qui assiomaticamente postulata sul piano storico, verrà poi riempita di contenuti politici aspramente polemici nel Convivio e nel De Vulgari. Essa è però già perfettamente delineata nel capitolo XXV della Vita Nuova ed avverte noi studiosi che il tratto più personale del pensiero linguistico di Dante consiste nella percezione della diglossia come elemento perverso della condizione umana, e storicamente obsoleto almeno nella società europea occidentale.

\section{L'idea di Grammatica dal Convivio al De Vulgari}

L’originalità del pensiero linguistico di Dante, sostanzialmente estraneo a tutte le tradizioni di pensiero, che egli attraversa liberamente estrapolando e contaminando, spiega da una parte il disinteresse degli immediati successori nei confronti della teoria esposta nel De Vulgari, e dall'altra le difficoltà che noi oggi troviamo nel ricostruirne le fonti. La polemica sulla influenza della grammatica speculativa, che infiamma da anni la bibliografia dantesca, è un sintomo di tali difficoltà, che dipendono da una oggettiva irriducibilità di quel pensiero a temi e prospettive tradizionali o condivise. Per cui credo che l'approccio migliore al pensiero dantesco consista nel metterne in luce gli spostamenti e le deviazioni, rispetto alle fonti, che pur esistono, e di peso, ma che sono utilizzate da Dante sempre in modo fortemente innovativo.

Partirò dalle considerazioni sulla Gramatica che lo scrittore svolge nel nono capitolo del primo trattato del De Vulgari. Dopo averla definita, al principio dell'opera, come una "locutio secundaria", rispetto al volgare lingua naturale, ${ }^{13}$ e dopo aver ricapitolato la storia del linguaggio a partire da Adamo e da Babele, il cui mito rappresenta la condanna divina alla instabilità linguistica, l'autore descrive l'inarrestabile processo di diversificazione idiomatica, fino alle differenze di linguaggio fra quartieri diversi della stessa città (per esempio Bologna). L'invenzione della grammatica, che non ha un inizio nel tempo ma risponde ad una esigenza antropologica di tipo metastorico, ${ }^{14}$ è iniziativa rifles-

13. (I I): «Locutio secundaria ... quam Romani gramaticam vocaverunt. Hanc quidem secundariam Greci habent et alii, sed non omnes. Ad habitum vero huius pauci perveniunt, quia non nisi per spatium temporis et studii assiduitatem regulamur et doctrinamur in illa» [un linguaggio di secondo grado, che i romani hanno chiamato grammatica. Questo linguaggio di secondo grado lo possiedono i greci e altri popoli, ma non tutti: pochi infatti arrivano a padroneggiarlo, dato che non riusciamo a farne nostre le regole a divenirne esperti se non col tempo e attraverso uno studio assiduo].

14. Svolgendo sul piano della antropologia le considerazioni dantesche sulla funzione della grammatica, potremmo dedurne che con la grammatica l'umanità esce dalla preistoria ed entra nella storia, poiché solo a partire dalla invenzione della scrittura, con cui la grammatica sostanzialmente si identifica, è possibile conservare la memoria dei fatti umani. 
sa ed artificiale, imposta all'umanità da questa naturale tendenza del linguaggio al mutamento:

De Vulgari I Ix 9-11. Non etenim admiramur si extimationes hominum qui parum distant a brutis, putant eandem civitatem sub inmutabili semper civicasse sermone, cum sermonis variatio civitatis eiusdem non sine longissima temporum successione paulatim contingat et hominum vita sit etiam ipsa sua natura brevissima Si ergo per eandem gentem sermo variatur, ut dictum est, successive per tempora, nec stare ullo modo potest, necesse est ut disiunctim abmotimque morantibus varie varietur, ceu varie variantur mores et habitus, qui nec natura nec consortio firmantur, sed humanis beneplacitis localique congruitate nascuntur. Hinc moti sunt inventores gramatice facultatis: que quidem gramatica nichil aliud est quam quedam inalterabilis lucutionis ydemptitas diversibus temporibus atque loci. Hec cum de comuni consensu multarum gentium fuerit regulata, nulli singulari arbitrio videtur obnoxia, et per consequens nec variabilis esse potest. Adinvenerunt ergo illam ne, propter variationem sermonis arbitrio singularium fluitantis, vel nullo modo vel saltim imperfecte antiquorum actingeremus autoritates et gesta, sive illorum quos a nobis locorum diversitas facit esse diversis.

[Non c'è da meravigliarsi se l'opinione di uomini poco lontani dai bruti ritiene che la stessa città abbia sempre vissuto la sua vita civile all'insegna di una lingua immutabile, dato che la variazione della lingua di una stessa città non si dà senza una lunghissima progressione di tempo, poco a poco, $\mathrm{e}$ dato che la vita umana è anche, per sua stessa natura, brevissima. Se dunque la lingua cambia entro una stessa gente, come si è detto, progressivamente nel tempo, e non può in alcun modo star ferma, necessariamente cambierà in modi diversi presso genti che abitano separate e distanti, come cambiano in modi diversi costumi e abitudini quando non sono stabilizzati né dalla natura né da un comune vincolo aggregante in loco. Da questo sono stati spinti coloro che hanno scoperto la facoltà della grammatica: la quale grammatica non è altro che una sorta di inalterabile identità della lingua attraverso tempi e luoghi diversi. Questa, poiché è stata regolata per consenso comune di molte genti, non appare esposta all'arbitrio individuale di nessuno, e di conseguenza non può neanche essere mutevole. L'hanno trovata, dunque, per evitare che, a causa del variare della lingua, fluttuante secondo l'arbitrio dei singoli, non potessimo in alcun modo attingere il sapere e la storia degli antichi, ovvero di coloro che la diversità dei luoghi rende diversi da noi].

Il senso è chiaro. La brevità della vita umana crea l'illusione di una continuità di linguaggio, nella stessa città, ed è quindi per un difetto di razionalità, proprio di uomini poco differenti dagli animali, che non viene avvertito il continuo processo di modificazione nel tempo e nello spazio delle lingue naturali, che il tempo trasforma come ogni altro comportamento o costume. Tale processo rese necessaria l'invenzione di uno strumento espressivo inalterabile che permettesse all'umanità di dialogare con gli antichi e comunicare con quanti sono distanti nello spazio. 
Di tale brano sono state indicate dagli studiosi del De Vulgari diverse fonti. Aristide Marigo ${ }^{15}$ e poi Maria Corti ${ }^{16}$ hanno ricondotto il pensiero di Dante al trattato sui Modi significandi di Boezio di Dacia, e concretamente a questo passaggio, che distingue fra la scoperta teorica della grammatica, opera del filosofi, e la sua applicazione normativa nei trattati, compito dei grammatici:

Ideo oportet grammaticae generationem fuisse per inventionem. Inventio autem grammaticae praecedit ipsam grammaticam. Ideo qui invenit grammaticam non fuit grammaticus. Item: aliquis est grammaticus in habendo grammaticam. Ipsam nullus habere potuit ante suam generationem. Ideo qui invenit eam non fuit grammaticus, sed erat philosophus proprias naturas rerum diligenter considerans.

Pier Vincenzo Mengaldo, ${ }^{17}$ Mirko Tavoni ${ }^{18}$ e Irène Rosier-Catach ${ }^{19}$ hanno addotto, soprattutto, due brani, uno dal De regimine principum, di Egidio Romano (I is 7) e l'altro dal Tesoro di Brunetto Latini versificato, più vicini al concetto dantesco di grammatica come strumento artificiale indipendente dalle lingue naturali:

Videntes enim philosophi nullum idioma vulgare esse completum et perfectum, per quod perfecte exprimere possent naturas rerum, et mores hominum, et cursus astrorum, et alia de quibus disputare volebant, invenerunt sibi quasi proprium idioma, quod dicitur latinum, vel idioma literale, quod constituerunt adeo latum et copiosum, ut per ipsum possent omnes suos conceptus sufficienter exprimere.

Sicome dicono i saggi, / ne la latina parlaura à diversi linguaggi: / uno linguaggio ànno l'Italici e un altro i Tedeschi, / e altro quelli d'Inghilerra e altro i Francieschi, / e tutti sono della parlaura latina comunemente. / E sì addiviene delli Ebrei e dei Greci, / che ànno fra lloro diversità di gente, / e diversi linguaggi ànno tra lloro / e perciò sono i Greci e li Ebrei / sicome latini costoro / e perciò i latini antichi e saggi / per rechare inn uno diversi linguaggi, / che s'intendesse insieme la gente, / trovaro la gramatica comunemente; / e così gli Greci e lli Ebrei in loro parlaura / trovaro loro gramatica e loro scritura. / Ciascuno trovò sue figure e sua maniera / quella delli Hebrei fu la primiera, / quella de li Greci fu la secondana, / quella de' Latini fu la diretana. / E li Ebrei, secondo che trovo per scritto, / trovarono la loro gramatica in Egitto; / i Greci, secondo che l'antica storia contiene, / trovarono la loro gramatica ind'Athene; / i Latini, secondo il loro ydioma, / trovarono la loro gramatica a Roma.

15. Aristide Marigo (a cura di), Dante, De Vulgari Eloquentia, Firenze: le Monnier, 1938.

16. Maria Corti, Dante a un nuovo crocevia, Firenze: Le Lettere, 1982, p. 38.

17. Pier Vincenzo Mengaldo (a cura di), De Vulgari Eloquentia, in Dante Alighieri, Opere, Minori II, Milano-Napoli: Mondadori, 2011.

18. Mirko Tavoni (a cura di), De Vulgari..., op. cit.

19. Irène Rosier-Catach (a cura di), Dante Alighieri, De l'eloquence en vulgaire, Paris: Fayard, 2011. 
A sua volta, Franco Lo Piparo ${ }^{20}$ ha osservato la analogia fra l'argomento dantesco circa le finalità della grammatica (comunicare con quanti sono distanti nel tempo e nello spazio) e quello addotto da Tommaso d'Aquino nel commento al Perihermeneias di Aristotele per giustificare l'invenzione della scrittura (De interpretatione, lib. 11.2 n. 2):

Est ergo considerandum quod circa primum tria proponit, ex quorum uno intelligitur quartum. Proponit enim scripturam, voces et animae passiones, ex quibus intelliguntur res. Nam passio est ex impressione alicuius agentis; et sic passiones animae originem habent ab ipsis rebus. Et si quidem homo esset naturaliter animal solitarium, sufficerent sibi animae passiones, quibus ipsis rebus conformaretur, ut earum notitiam in se haberet; sed quia homo est animal naturaliter politicum et sociale, necesse fuit quod conceptiones unius hominis innotescerent aliis, quod fit per vocem; et ideo necesse fuit esse voces significativas, ad hoc quod homines ad invicem conviverent. Unde illi, qui sunt diversarum linguarum, non possunt bene convivere ad invicem. Rursum si homo uteretur sola cognitione sensitiva, quae respicit solum ad hic et nunc, sufficeret sibi ad convivendum aliis vox significativa, sicut et caeteris animalibus, quae per quasdam voces, suas conceptiones invicem sibi manifestant: sed quia homo utitur etiam intellectuali cognitione, quae abstrahit ab hic et nunc; consequitur ipsum sollicitudo non solum de praesentibus secundum locum et tempus, sed etiam de his quae distant loco et futura sunt tempore. Unde ut homo conceptiones suas etiam his qui distant secundum locum et his qui venturi sunt in futuro tempore manifestet, necessarius fuit usus scripturae.

Di questi brani quello indubbiamente più pertinente al testo di Dante è il commento tomista, che indica come ragioni per l'invenzione della scrittura le stesse indicate da Dante per l'invenzione della grammatica, e cioè la necessità degli uomini di manifestare i propri concetti «a distanza». Lungi dal trattarsi di una analogia estrinseca, la coincidenza fra l'argomento di Dante e quello di Tommaso mostra come il primo restringa il concetto di grammatica, esaltandone la artificialità, utilizzando le nozioni del secondo relative alla scrittura. Il che certo mostra, come vuole Lo Piparo, la differenza di Dante rispetto alla teoria dei modisti (per i quali la grammatica è implicita nel linguaggio naturale, di cui costituisce la struttura profonda). ${ }^{21} \mathrm{Ma}$ indica anche, dall'altra

20. Franco Lo Piparo, Sign and Grammar in Dante. A Non-Modistic Laguage Theory, in Paolo RAmat et al. (ed.), The History of linguistics in Italy, Amsterdam / Philadephia: John Benjamins Publishing Company, I986, p. 9-IO.

21. Lo Piparo mostra inoltre come l'espressione "grammatice facultas» appartenga al lessico dell'insegnamento scolastico, nel quale indicava le abilità elementari della lettura e la scrittura (op. cit., p. 9). Negli atti del concilio lateranense del 1215 si prescrive che un maestro "clericos eiusdem ecclesiae aliosque scholares pauperes gratis instrueret in grammaticae facultate ac aliis». Il testo è citato in Giuseppe Manacorda, Storia della scuola in Italia. Il medio evo, Firenze: Le Lettere, 1980 (1914), I, p. 72. Lo studio di Manacorda è estremamente interessante anche per altri aspetti. In particolare, lo storico mette bene in luce da una parte il monopolio dell' istruzione perseguito con successo dalla chiesa a partire dal papa Gregorio VII, dall'altra il diffuso peccato di simonia consistente nell'infeudamento 
parte, che la riflessione dantesca è profondamente radicata in una linea di pensiero aristotelico-tomista. Il che ci apre uno spiraglio forse rivelatore sul percorso teorico di Dante.

Come è noto, il rapporto semiotico biunivoco fra linguaggio e pensiero è il fondamento della logica aristotelica, che identifica le categorie del pensiero con funzioni sintattico-grammaticali (soggetto e predicato). La riflessione su tale equivalenza ed il suo approfondimento metodologico, potremmo dire: la sua considerazione estensiva, stimola un approccio speculativo, prima in Boezio e nei suoi commenti alla logica di Aristotele e poi nella filosofia del linguaggio nel secolo XIII, che determina il superamento della più tradizionale concezione descrittiva e normativa della grammatica, operante nei manuali destinati all'insegnamento. ${ }^{22}$ Sebbene non interessato in particolare ai «modi significandi", Tommaso svolge un ruolo decisivo nel riorientare in senso speculativo la considerazione del linguaggio, poiché nella sua gnoseologia la parola (il verbum) svolge una capitale funzione mediatrice nel processo di elaborazione mentale del concetto. È un tema caratteristico di Tommaso e che suscitò vive controversie, poiché la ragione astratta del concetto veniva subordinata alla sua fomulazione verbale (il verbum mentis), necessaria perché il contenuto della razionalità si attualizzi nella mente. Come è stato messo in evidenza dagli studiosi della filosofia medievale del linguaggio, il verbum mentis di Tommaso riprende un concetto agostiniano, quello di verbum cordis, ridefinendolo a partire dalla teoria aristotelica della conoscenza. Si tratta cioè di una parola interna e necessaria al processo di astrazione che dal fantasma sensibile deduce l'idea. ${ }^{23}$

Come ha mostrato Mirko Tavoni nel suo commento al De Vulgari, la dipendenza di Dante da Tommaso, nella teorizzazione relativa al segno linguistico, è sostanziale. ${ }^{24}$ Credo che anche in rapporto alla idea di grammatica

della «licentia docendi», contro cui Dante scaglia il suo anatema in Conv. I Ix. Attualissime e pienamente vigenti sono le conclusioni dello studioso relativamente a Dante e al suo rapporto con la cultura clericale: «Dante, pio e devoto, che s'india nei cieli, Dante dotto, filosofo e teologo, benché laico, Dante cittadino, ecco l'uomo che compendia gli effetti di una politica scolastica, opera costante della Chiesa. Ma rugge nell'animo religioso di Dante un fiero spirito civile ed antipapale; è un segnale dei tempi nuovi! La borghesia, il laicato, vorrà ora una scuola sua, e l'avrà, contrapponendola a quella chiesastica, d'onde esso medesimo è uscito» (I, p. 94).

22. «I modi significandi (sia essenziali che accidentali) si sovrappongono alla significazione lessicale, come consignificazione o connotazione, secondo uno schema triangolare che ricalca quello tradizionalmente derivato dal De interpretazione per il rapporto tra vox-conceptus-res", Costantino Marmo, "Grammatica e semantica dei modisti: fonti e chiavi di lettura", Studi filosofici, n. 29, 2006, p. 323-335 (citazione p. 327) .

23. Cfr. Alain De Libera, La querelle des universaux, Paris: Seuil, 1996, p. 273-276 e Claude Panaccio, Le discours intérieur de Platon à Guillaume d'Ockham, Paris: Seuil, 1999, p. $179-186$.

24. Mirko Tavoni l'ha messa in evidenza in relazione, fra l'altro, alla definizione di segno linguistico, in De Vulgari I III 3, nella quale il segno viene concepito non come elemento nucleare della lingua ma come locutio, cioè linguaggio: «Hoc equidem signum est ipsum 
la prospettiva tomista ha offerto a Dante spunti decisivi. Per apprezzarli bisogna soffermarsi brevemente sulla articolazione del commento di Tommaso al Perihermeneias che abbiamo appena considerato. Qui Tommaso interpreta le equivalenze semiotiche di Aristotele con una sorprendente ed originale apertura di orizzonti filosofici. Innanzitutto giustifica la necessità del soggetto di indicare verbalmente le passioni dell'anima attraverso la natura sociale e politica dell'essere umano, la qual cosa determina un sostanziale spostamento del problema semantico: ciò che essenzialmente importa del significato delle parole non è il rapporto denotativo con le cose (o con il pensiero delle cose), come vogliono i logici e lo stesso Aristotele, ma il rapporto comunicativo fra gli uomini. Si tratta di "conceptiones unius hominis innotescere aliis» (Dante scrive, I III 2: «ad communicandas inter se conceptiones suas», ma in I II 3, relativamente agli angeli, "alter alteri ... innotescit» il proprio concetto), poiché, come leggiamo nel commento alla Politica di Aristotele, «loquutio humana significat quid est utile et quid nocivum» (I 1 29). ${ }^{25}$ In virtù di tale funzione eminentemente politica, convivono male, aggiunge Tommaso nel passo del commento al Perihermeneias citato sopra, uomini di lingue diverse. Ma più significativa ancora, in Tommaso, è la attribuzione al linguaggio dei processi astrattivi che consentono all'essere umano di superare l'hic et nunc della percezione immediata. Se si trattasse solo di indicare con i suoni della voce le passioni dell'anima prodotte dalla sensibilità, non sarebbe necessario il linguaggio articolato: vediamo infatti che alcuni animali hanno questo tipo di linguaggio. Gli uomini, invece, hanno anche cognizioni intellettuali, che non dipendono dalla immediatezza percettiva, e per esprimere le quali è necessario un linguaggio concettualmente articolato, dal quale dipende poi l'invenzione della scrittura. Si osservi, in questo passo del Commento alle Sentenze (lib. 2 d.

subiectum nobile de quo loquimur: nam sensuale quid est, in quantum sonus est; rationale vero, in quantum aliquid significare videtur ad placitum» [Questo segno è precisamente il soggetto nobile di cui parliamo: il quale, infatti, è qualcosa di sensibile in quanto è suono, ed è qualcosa di razionale in quanto, evidentemente, significa in modo arbitrario]. Leggiamo, per esempio, nel De veritate (9 4 4): «Omnis locutio est per aliquod signum» e nel commento alle Sentenze (Super sententiis II, 1123 2): «in omni locutione oportet esse aliquod signum, quod mentis occultum conceptum exprimat». Ma si vedano anche le note relative a I xvi. Nella stessa direzione Karl Otto ApeL, Lidea di lingua nella tradizione dell'umanesimo da Dante a Vico, Bologna: Il Mulino, 1975, p. 136.

25. Ma vale la pena di leggere tutto il passo per rendersi conto di come Tommaso identifichi la funzione comunicativa del linguaggio (quella che per Dante è l'unica sostanzialmente rilevante) con la natura politica dell'essere umano: «loquutio humana significat quid est utile et quid nocivum. Ex quo sequitur quod significet iustum et iniustum. Consistit enim iustitia et iniustitia ex hoc quod aliqui adaequentur vel non aequentur in rebus utilibus et nocivis. Et ideo loquutio est propria hominibus; quia hoc est proprium eis in comparatione ad alia animalia, quod habeant cognitionem boni et mali, ita et iniusti, et aliorum huiusmodi, quae sermone significari possunt. Cum ergo homini datus sit sermo a natura, et sermo ordinetur ad hoc, quod homines sibiinvicem communicent in utili et nocivo, iusto et iniusto, et aliis huiusmodi; sequitur, ex quo natura nihil facit frustra, quod naturaliter homines in his sibi communicent. Sed communicatio in istis facit domum et civitatem. Igitur homo est naturaliter animal domesticum et civile». 
11 q. 2 a. 3 co.), di cui si avverte chiaramente l'eco nella definizione dantesca del segno linguistico, con quanta precisione Tommaso identifichi le componenti interiori della esperienza umana («interiores conceptus mentis») con i suoi aspetti intellettuali («ratio intelligibilis»), che in quanto ordinati alla comunicazione assumono una forma linguistica («verbum cordis»):

Est enim aliquid in homine quod alius homo de ipso naturaliter percipere potest, ut ea quae exterioribus sensibus subjacent; aliquid vero quod videri non potest, sicut interiores conceptus mentis. Species ergo conceptae interius, secundum quod manent in simplici conceptione intellectus, habent rationem intelligibilis tantum: secundum autem quod ordinantur ab intelligente ut manifestandae alteri, habent rationem verbi, quod dicitur verbum cordis; secundum autem quod aptantur et quodammodo ordinantur signis exterius apparentibus, si quidem sunt signa ad visum, dicuntur nutus; si vero ad auditum, dicitur proprie locutio vocalis: hi enim duo sensus disciplinabiles sunt.

In questi testi di Tommaso sono due gli elementi che, credo, hanno influito potentemente sulla teorizzazione di Dante. Il primo è la esigenza di razionalità che è immediatamente connessa al significato delle parole. Ciò vuol dire che le funzioni intellettuali della mente sono coinvolte nel linguaggio per natura ed anteriormente ad ogni attività psichica successiva e secondaria. L'esercizio della razionalità umana è già implicito nel fatto che l'essere umano parla, per tanto esso si dispiega immediatamente nell'uso spontaneo e naturale del linguaggio. La scrittura è conseguenza di tale istanza razionale ed interviene, in un secondo momento, per garantire la comunicazione con chi è lontano o assente. Notevolissima è, su questo punto essenziale, la differenza rispetto ad Agostino (al quale spesso la linguistica di Dante viene ricondotta): mentre in Agostino il verbum cordis, la parola del cuore, si impoverisce nella sua semiosi verbale, cioè perde la sua energia illuminativa, poiché il linguaggio proferito o pensato afferisce esclusivamente alla sensibilità, ${ }^{26}$ in Tommaso il segno verbale è «verbum mentis» necessario alla formazione del concetto. Esso ha dunque, contrariamente alla svalutazione agostiniana, una natura nitidamente speculativa, anche se su un piano diverso da quello esplorato dai modisti: esso è molto più concettuale che grammaticale. Il secondo elemento che deve essere attentamente valutato della rilettura tomista di Aristotele è l'aggancio della funzione comunicativa del linguaggio alla natura politica dell'essere umano: "communicatio facit domum et civitatem», scrive Tommaso nel commento alla Politica qui citato in nota, e nel commento al Perihermenias:

26. «La parola esterna, come anche la parola esterna solo riprodotta nell'interiorità, è legata a una lingua determinata. Il fatto che in ogni linguaggio il verbum sia espresso in modo diverso significa soltanto, tuttavia, che esso non puo mostrarsi, nel suo vero essere, nella lingua umana. Dice Agostino, con una svalutazione tutta platonica dell'apparenza sensibile: non dicitur, sicuti est, sed sicut potest videri audirive per corpus. La parola "vera", il verbum cordis, è del tutto indipendente da questa apparenza. Non è né prolativum né cogitativum in similitudine soni» (Hans Georg Gadamer, Verità e metodo, Milano: Bompiani, 1989, p. 482). 
«qui sunt diversarum linguarum, non possunt bene convivere ad invicem». La civitas è possibile solo dove esiste unità di lingua, il che fa del linguaggio una esperienza ed una pratica immediatamente vincolate alle strutture politiche, la cui capacità di aggregare gli individui dipende innanzitutto dalla fluidità della circolazione linguistica. ${ }^{27}$

Provo ora a verificare la pertinenza di questi assiomi ad una delle grandi cruces dantesche, e cioè la contraddizione in cui sembra cadere lo scrittore quando afferma nel Convivio che il latino è più nobile del volgare, e, nel $D e$ Vulgari, che il volgare è più nobile del latino. L'ipotesi che io qui proporrei è che si tratta non di una contraddizione, ma di fasi diverse di un percorso intellettuale che ha, come suo decisivo punto di svolta, la meditazione sui brani di Tommaso che stiamo analizzando (e su altri dello stesso tenore). La differenza più clamorosa fra le due opere, relativamente alla teorizzazione sul linguaggio, è infatti, a mio avviso, la sostanziale assenza di una istanza razionalistica nel $1^{\circ}$ trattato del Convivio, e, al contrario, l'appello sistematico al razionalismo strutturale del linguaggio nel De Vulgari, razionalismo che Dante ha appreso da Tommaso, dalla sua teoria generale del verbum mentis $\mathrm{e}$, più concretamente, dei identificazione di significato verbale e concetto mentale che abbiamo appena considerato. Nel Convivio il volgare è meno nobile perché non ha prestigio, e deve essere preferito al latino non per motivi di razionalità intrinseca, ma per motivi culturali e politici (ed in particolare per il tradimento degli chierici, che hanno prostituito la letteratura). Il rapporto latino / volgare non è analizzato dal punto di vista dei contenuti intellettuali mobilitati dalle due lingue (che anzi sarebbe tutto a vantaggio del latino in quanto arte), ma bensì da quello dei gruppi sociali che ne fanno uso: da una parte i chierici, che hanno il monopolio della cultura e nessun rapporto con la società civile, dall'altra i laici, perfettamente integrati nella società civile ma privi di cultura (ad alto livello). Il latino è più nobile per la sua perfezione grammaticale, per cui è logico che ad esso, in quanto prodotto $\mathrm{d}$ 'arte, cioè di studio, spetti un contenuto intellettuale più trasparente $\mathrm{e}$ quindi maggiore nobiltà. Il volgare, però, in quanto lingua propria, cioè strumento espressivo della comunità familiare e sociale, è destinato a sostituirlo, poiché la società civile ha maturato valori che il latino, lingua separata, sarebbe incapace di esprimere. Il volgare è quindi preferibile al latino non per sé, ma relativamente a mutate condizioni storiche che, come Dante aveva già intuito nella Vita Nuova, lo rendono modernamente necessario su un piano che non è più semplicemente poetico, come nel libello giovanile, ma ampiamente culturale e politico.

Quando intraprende la stesura del De Vulgari, Dante ha scoperto, invece, grazie a Tommaso, che le funzioni elevate del linguaggio, quelle che ne fanno

27. Mette in evidenza tale aspetto sostanziale della riflessione dantesca sul linguaggio, ed anzi lo fa coincidere con la stessa genesi del De Vulgari, Mirko Tavoni nella sua edizione del trattato (p. 1068-1069 e 1139-1140). 
uno strumento espressivo al servizio della razionalità, non sono secondarie o artificiali, ma implicite nelle sue componenti semantiche e concettuali, che ovviamente sono già operative nel linguaggio orale, quindi nel volgare inteso come lingua materna. Ed infatti la cellula originaria e fondamentale del linguaggio umano, cioè il segno, viene immediatamente definito come portatore di razionalità (sul versante del significato: I III 2-3), ed è tale strutturale razionalità del segno linguistico che si proietta sul linguaggio naturale nel suo insieme nobilitandolo rispetto ad ogni altro codice secondario o artificiale. Si badi bene, tale strutturale e originaria nobiltà del volgare è operativa nel linguaggio inteso come locutio, cioè come naturale facoltà espressiva e comunicativa, non in questo o quell' idioma volgare, degenerato, quasi sempre, ${ }^{28}$ perché abbandonato alla logica dispersiva del mutamento spaziotemporale, senza una cultura letteraria ed uno stato che lo coltivino e preservino. In sé razionale, il linguaggio umano (la locutio) vanifica la sua nobile funzione se non dispone di una cultura letteraria (una eloquentia) che ne attualizzi le potenzialità. E si tratta di una eloquentia non specialistica e separata (come quella dei chierici) ma naturale e socialmente diffusa, infatti ad essa tendono anche le donne e i bambini.

La scrittura, cioè l'arte grammaticale, non aggiunge nulla, sul piano intellettuale, al linguaggio; è solo uno strumento ulteriore, una artificiale tecnologia della parola che garantisce la comunicazione a distanza. Di qui la svalutazione antropologica della scrittura e la parallela scoperta della funzione formativa che ha nella mente la lingua materna, la cui nobiltà dipende dalla sua universalità: tutti gli uomini hanno un linguaggio come tutti gli uomini hanno una razionalità, l'uno e l'altro sono connaturati alla specie umana. Altrettanto non si può dire della grammatica, cioè della scrittura, che solo alcuni imparano, con grande sforzo.

$\grave{\mathrm{E}}$ appunto questa la scoperta che Dante rivendica nel trattato latino. Seguendo le indicazioni teoriche di Tommaso, ed applicandole al rapporto diglossico latino-volgare, egli può proclamare un principio o una legge che, effettivamente, mai nessuno aveva formulato, e cioè la superiore dignità ontologica del volgare, in quanto lingua orale e primaria, rispetto al latino, che è lingua scritta e secondaria. Tale scoperta determina, credo, anche la decisione di scrivere il trattato linguistico in latino e non in italiano, perché la sua funzione non è divulgativa, come lo è quella del Convivio, ma rivelativa, e deve essere quindi presentata e sottoposta innanzitutto alla comunità scientifica. Quando Dante annuncia, nel $1^{\circ}$ Tratattato del Convivio, «uno libello ... di Volgare Eloquenza», non pensa ancora ad uno scritto in latino, perché la sua teoria del volgare è ancora largamente debitrice di una concezione tradiziona-

28. Le eccezioni sono quelle descritte nel De Vulgari come esperienze di poesia che tendono al volgare illustre. Sulla terminologia linguistica usata da Dante nel De Vulgari, si veda Mirko TAvonI, "Contributo all'interpretazione di De Vulgari Eloquentia I 1-9", Rivista di letteratura italiana, V, n. 3, 1987, p. 385-453. 
le, prescientifica, del linguaggio. ${ }^{29}$ Infatti nel De Vulgari il testo sarà definito non libellus ma opus, poiché la sua finalità è doctrinam tractare (o tradere)..$^{30}$

Più che di una palinodia si tratta di una scoperta teorica che interviene durante la stesura del Convivio. Le argomentazioni che, nel trattato italiano, immediatamente precedono l'annuncio del futuro libello sono infatti proprio quelle che abbiamo letto ora nel De Vulgari che spiegano le cause della invenzione della grammatica, e cioè la mutabilità del linguaggio che rende necessario uno strumento stabile, come il latino (I v 7-10):

Ché, primamente, [il volgare] non era subietto ma sovrano, e per nobilità e per vertù e per bellezza. Per nobilità, perché lo latino è perpetuo e non corruttibile, e lo volgare è non stabile e corruttibile. Onde vedemo ne le scritture antiche de le comedie e tragedie latine, che non si possono transmutare, quello medesimo che oggi avemo; che non aviene del volgare, lo quale a piacimento artificiato si transmuta. Onde vedemo ne le cittadi d'Italia, se bene volemo agguardare, da cinquanta anni in qua molti vocabuli essere spenti e nati e variati; onde se 'l picciol tempo così transmuta, molto più transmuta lo maggiore. Sì ch'io dico che se coloro che partiron d'esta vita già sono mille anni tornassero a le loro cittadi, crederebbero la loro cittade essere occupata da gente strana, per la lingua da [lla] loro discordante. Di questo si parlerà altrove più compiutamente in uno libello ch'io intendo di fare, Dio concedente, di Volgare Eloquenza.

Mentre qui il requisito della nobiltà dipende dal tempo, per cui è più nobile ciò che dura di più, nel De Vulgari esso dipende dalla razionalità, per cui è più nobile ciò che è più razionale. La nuova prospettiva teorica, per cui la grammatica-scrittura implica non un incremento di razionalità del linguaggio, ma solo la sua estensione ad ambiti comunicativi più ampi, interviene dopo la stesura dei primi trattati del Convivio e determina anche il mutamento di lingua, rispetto al progetto originario: latino e non volgare perché si tratta di un'opera di ricerca scientifica originale e non di divulgazione. Maria

29. Le due testimonianze più antiche sul trattato (Villani Cronica X 136 e Boccaccio Trattatello XXVI) deducono il titolo direttamente dal Convivio («fece uno libretto che l'intitola de vulgari eloquentia», "compuose uno libretto in prosa latina il quale egli intitolò De Vulgari eloquentia»), trascurando il diverso rango che l'opera progettata aveva acquisito in corso di stesura.

30. I XIX 2: «Et quia intentio nostra, ut polliciti sumus in principio huius operis, est doctrinam de vulgari eloquentia tradere»; II I 1: «Sollicitantes iterum ingenii nostri et ad calamum frugi operis redeuntes». Ma l'intenzione "dottrinale» dell'opera viene dichiarata significativamente all'inizio (I I 2), quando Dante scrive: «Sed quia unamquamque doctrinam oportet non probare, sed suum aperire subiectum, ut sciatur quid super quod illa versatur...». La impossibilità o la inutilità di probare i principia delle diverse scienze particolari viene così enunciata nella prima Quaestio (art. 6 ad 2) della Summa Theologiae di Tommaso d'Aquino: «aliarum scientiarum principia vel sunt per se nota, et probari non possunt, vel per aliquam rationem naturalem probantur in aliqua alia scientia» (i principi della teologia, invece, dipendono dalla rivelazione). Facendo suo tale assioma, Dante implicitamente rivendica il rango di scienza alla eloquenza volgare. Ed in ciò, appunto, consiste la rivoluzione culturale da lui auspicata. 
Corti sostenne persuasivamente che il De Vulgari fu composto in un periodo successivo alla redazione dei primi tre trattati del Convivio e prima di scrivere il IV. ${ }^{31} \mathrm{E}$ in effetti il quarto trattato del Convivio ha la struttura di una "quaestio de nobilitate», cioè di un trattato filosofico al suo livello più alto, mentre i primi tre hanno un andamento più enciclopedico e divulgativo che speculativo. È più che probabile, quindi, che proprio attraverso il De Vulgari maturi in Dante una coscienza autoriale già dottrinaria, sperimentata prima in latino e poi estesa al volgare nell'ultimo trattato del Convivio. La stessa idea di nobiltà, intorno a cui ruota il IV trattato, sembra implicare la superiore dignità della cultura laica e volgare già dimostrata nel trattato latino attraverso la superiorità del linguaggio naturale su quello artificiale.

Fra gli argomenti che, nel primo del Convivio, vengono addotti per giustificare l'abbandono del latino come lingua letteraria e l'adozione del volgare, l'unico che faccia appello alla razionalità è quello che troviamo nel capitolo XI, uno «speziale capitolo» nel quale viene denunciata la infamia «de li malvagi uomini d'Italia che commendano lo volgare altrui e lo loro proprio dispregiano». Delle cinque «abominevoli cagioni» che spingono gli Italiani a preferire le lingue altrui interessa qui la prima, e cioè la «cechitade di discrezione». Il brano merita di essere analizzato con una certa attenzione:

I xi 3-10. Sì come la parte sensitiva de l'anima ha suoi occhi, con li quali aprende la differenza de le cose in quanto elle sono di fuori colorate, così la parte razionale ha suo occhio, con lo quale aprende la differenza de le cose in quanto sono ad alcuno fine ordinate: e questo è la discrezione. E sì come colui che è cieco de li occhi sensibili va sempre secondo che li altri giudicando lo male e lo bene, così colui che è cieco del lume della discrezione sempre va nel suo giudicio secondo il grido, o diritto o falso; onde, qualunque ora lo guidatore è cieco, conviene che esso e quello, anche cieco, ch'a lui s'appoggia, vegnano a mal fine. Però è scritto che "l cieco al cieco farà guida, e così cadranno ambedue nella fossa». Questa grida è stata lungamente contro a nostro volgare per le ragioni che di sotto si ragioneranno apresso di questa. E li ciechi sopra notati, che sono quasi infiniti, con la mano in su la spalla a questi mentitori, sono caduti ne la fossa de la falsa oppinione, de la quale uscire non sanno. De l'abito di questa luce discretiva massimamente le populari persone sono orbate; però che, occupate dal principio de la loro vita ad alcuno mestiere, dirizzano sì l'animo loro a quello per [la] forza de la necessitate, che ad altro non intendono. E però che l'abito di vertude, sì morale come intellettuale, subitamente avere non si può, ma conviene che per usanza s'acquisti, ed ellino la loro usanza pongono in alcuna arte e a discernere l'altre cose non curano, impossibile è a loro discrezione avere. Per che incontra che molte volte gridano Viva la loro morte, e Muoia la loro vita, pur che alcuno cominci; e questo è pericolosissimo difetto ne la loro cechitade. Onde Boezio giudica la populare gloria vana, perché la vede sanza discrezione Questi sono da chiamare pecore, e non uomini; ché se una pecora si gittasse da una ripa di mille passi, tutte l'altre

31. Maria Conti, La felicità mentale. Nuove prospettive per Cavalcanti e Dante, Torino, Einaudi, 1983, p. 123-145. 
l'anderebbero dietro; e se una pecora per alcuna cagione al passare d'una strada salta, tutte l'altre saltano, eziandio nulla veggendo da saltare. E io ne vidi già molte in uno pozzo saltare per una che dentro vi saltò, forse credendo saltare uno muro, non ostante che 'l pastore, piangendo e gridando, con le braccia e col petto dinanzi a esse si parava.

Cieche, ossia non assistite dal lume della razionalità, sono le «populari persone» le quali, per il fatto di essere occupate in una qualunque attività lavorativa o professionale, si lasciano guidare da una falsa opinione circa la bellezza e l'utilità del proprio volgare, sottovalutate a vantaggio di lingue altrui (come potrebbero essere il provenzale e il francese, effettivamente usate in Italia da poeti e scrittori). Tali persone, quasi infinite di numero, sono comparabili ai ciechi del Vangelo oppure ad un gregge di pecore, e cadono ignare nella fossa mortale di questa "falsa oppinione». L'appello alla razionalità del linguaggio è, qui, abbastanza estrinseco poiché di esso non tocca, come nel De Vulgari, la natura espressiva o comunicativa. La razionalità di cui viene lamentata l'assenza nelle «populari persone» è la coscienza della fondamentale funzione civile della lingua propria all'interno della comunità. Ci sono, tuttavia, immagini e temi che saranno ripresi nel De Vulgari, ma utilizzati in modo significativamente distinto. Innanzitutto la cecità delle «populari persone». Il trattato latino si apre proprio con questa immagine (I 1):

volentes discretionem aliqualiter lucidare illorum qui tanquam ceci ambulant per plateas, plerunque anteriora posteriora putantes

[volendo in qualche modo illuminare il discernimento di quanti vagano come ciechi per le piazze, per lo più credendo di avere dietro le spalle quello che hanno davanti agli occhi].

È chiara, da un trattato all'altro, la ripresa del tema evangelico. Ma si osservi come cambia l'atteggiamento dello scrittore: nel Convivio la cecità delle "populari persone» viene solo denunciata, nel De Vulgari, invece, Dante rivendica per sé e per l'opera che sta scrivendo la missione di illuminare e redimere chi brancola nel buio dell'ignoranza. Anche il finale del primo trattato del Convivio fa appello alla luce che traerà con sé il volgare (I XIII):

Questo sarà luce nuova, sole nuovo lo quale surgerà là dove l'usato tramonterà, e darà lume a coloro che sono in tenebre e in oscuritade per lo usato sole che a loro non luce.

$\mathrm{Ma}$, anche qui, viene preannunciata una palingenesi oggettiva di cui il poeta è solo banditore, non protagonista ed autore. Ė evidente il salto di qualità che lo scrittore presume di aver realizzato all'altezza del De Vulgari: non si tratta più, semplicemente, di divulgare o proclamare, ma di illuminare, grazie ad una scoperta destinata a rivoluzionare la cultura linguistica dell'umanità.

È vero, d'altra parte, che anche nel brano del Convivio sui «malvagi uomini d'Italia» Dante allude a se stesso come a colui che è investito di una profe- 
tica missione redentiva. Lo vediamo nella immagine finale delle pecore che si buttano in un pozzo, "non ostante che 'l pastore, piangendo e gridando, con le braccia e col petto dinanzi [a esse] si parava». Non c’è dubbio che nel pastore Dante ha proiettato se stesso, la cui missione consiste appunto nell'aprire gli occhi degli Italiani, evitando che precipitino nell'abisso dell'ignoranza e delle lotte fratricide. ${ }^{32}$ Lo fa però in modo indiretto, forse allegorico. Nel De Vulgari, invece, la propria coscienza di auctor raggiunge i vertici della gerarchia del sapere. Non più «ai piedi di coloro che seggiono a la beata mensa», egli è ora, semmai, il capotavola, che si accinge a dispensare ai dotti un sapere finora ignorato.

Il brano del Convivio ci mostra poi un ulteriore aspetto, di estremo rilievo nella teoria dantesca del linguaggio, accanto a quello filosofico e letterario, e cioè quello politico, anch'esso dipendente dalle straordinarie suggestioni della rilettura aristotelica di Tommaso. La definizione di razionalità che Dante qui propone (cioè la capacità di vedere «la differenza de le cose in quanto sono ad alcuno fine ordinate»), tradotta nel suo contrario come cecità politica, significa la incapacità del popolo cittadino, diviso in arti e corporazioni, di vedere il bene comune e di subordinare ad esso gli interessi di parte: schiave del proprio egoismo corporativo, le «populari persone» non comprendono che ogni singolo mestiere ha senso e può sussistere esclusivamente nella impresa comune rappresentata dall'insieme della società civile, della quale il volgare è cemento unificante. La divisione sociale del lavoro, che a Firenze si è immediatamente proiettata negli ordinamenti politici del comune, cioè nel sistema delle Arti, e che quindi ha dato luogo agli endemici conflitti cittadini di cui lui stesso è stato vittima, ha come deleteria conseguenza la impossibilità di percepire i legami di solidarietà fra i gruppi, la convergenza dei singoli mestieri, e degli interessi relativi, verso un unico fine che è il benessere dalla città e della collettività. In tale mancanza di visione d'insieme consiste la «cechitade delle populari persone». L'acutezza del pensiero del poeta qui è, direi, clamorosa. Come ha fatto, o sta per fare, nella canzone Doglia mi reca e come farà nel IV Trattato del Convivio, che denunciano gli effetti perversi della nuova economia monetaria sul tessuto comunitario, qui Dante denuncia gli effetti disgreganti di una economia che soggioga la politica, sostituendo gli interessi dei gruppi al benessere comune. Sono intuizioni che nascono da motivazioni radicalmente personali, e cioè il feroce risentimento anticomunale degli anni dell'esilio, quando ogni possibilità di rientro a Firenze è preclusa, ma che colgono sul

32. Si osservi come il sintagma "con le braccia e col petto» risuoni nei versi dedicati ad un personaggio della Commedia nei confronti del quale l'ammirazione di Dante è massima (Farinata): «ed el s'ergea col petto e con la fronte» (Inf. X 35). Se si considera che anche l'altro sintagma, "piangendo e gridando", verrà riutilizzato a proposito del personaggio di Cavalcante: "piangendo ... gridò" (Inf. X 58-67), e che il brano del Convivio denuncia la "cechitade del lume della discrezione», potremo agevolmente concludere che in esso aleggia l'ombra del "primo amico», anch' egli coinvolto, ma certo su un altro piano e per altri motivi, nella polemica linguistica sulla difesa del volgare proprio. 
nascere le linee di tendenza della società moderna, che significano la liquidazione del modello solidaristico dello "status in ordine triplex» (e la sua sostituzione con modelli dicotomici basati sulla lotta di classe). Ma ciò che soprattutto interessa qui è la vincolazione del sentimento collettivo di solidarietà sociale con l'amore del volgare proprio. Qui è infatti già operante il modello teorico tomista, che nell'unità di lingua fonda il sentimento della civitas. Esso è però ancora imperfettamente calibrato sulla realtà sociale e politica del comune fiorentino. Non si vede infatti perché la divisione del lavoro dovrebbe nuocere all'attaccamento delle persone alla propria lingua. Sembrerebbe semmai il contrario, poiché la difesa egoistica dei propri interessi economici e della propria attività produttiva (il mestiere) dovrebbe naturalmente portare gli esseri umani a rivendicare ciò che appartiene loro anche sul piano del linguaggio (il «volgare proprio»).

Il nesso fra la cecità delle "populari persone», dovuta alla parzialità e all'egoismo dei mestieri, e l'incomprensione della funzione del linguaggio nella civitas viene invece perfettamente descritto nel De Vulgari, dove il mito babelico è riletto proiettando sul racconto biblico l'esperienza comunale e la divisione in arti della cittadinanza. Il castigo divino della superbia umana non si limita, infatti, a confondere la lingua dei costruttori della torre individualmente considerati, ma segue esattamente i confini dei diversi mestieri, in modo tale che ad ogni gruppo di operai resta un proprio linguaggio: la divisione sociale del lavoro, già bollata di ignoranza e cecità nel Convivio, viene ora ulteriormente condannata come effetto di perversione e superbia nel $D e$ Vulgari, che ne mette in evidenza le tendenze centrifughe sul piano del linguaggio. ${ }^{33} \mathrm{E}$ si osservi come Dante ridefinisca, sul piano teorico, la funzione comunicativa del linguaggio riscrivendola all'interno del modo di produzione economico: posta una divisione funzionale e gerarchica del lavoro (semplice quanto si voglia, come potrebbe essere quella di un cantiere edilizio, ma pur sempre rappresentativa del sistema delle Arti nel comune fiorentino), la funzione comunicativa politicamente rilevante è quella che si stabilisce fra gruppi omogenei di lavoratori, non fra parlanti isolati. La genialità di tale prospettiva teorica dipende proprio dalla sua improbabilissima verifica fattuale: forzando il concetto di comunicazione linguistica, Dante ne mette in luce i legami profondi e sostanziali con la struttura economico-politica all'interno della quale una lingua è operativa. Laddove gli interessi economici di parte surrogano o comunque si sovrappongono all'interesse comune disgregando la società civile, gli uomini credono di parlare la stessa lingua, in realtà sono così estranei gli uni agli altri come dovevano esserlo i costruttori di Babele.

Rivelatore della prospettiva immediatamente politica della teoria dantesca del linguaggio è il confronto fra l'interpretazione di Babele del De Vulgari

33. Anche su questo punto è illuminante la lettura di Maria ConTI, «Dante e la Torre di Babele», in ID., Il viaggio testuale, Torí, Einaudi, 1978, p. 245-256. 
(I vir 6-7) e quelle della General Estoria (1.43b24) e dello Speculum Historiale (24a):

D.V.E. Siquidem pene totum humanum genus ad opus iniquitatis coierat. Pars imperabant, pars architectabantur, pars muros moliebantur, pars amysibus regulabant, pars trullis linebant, pars scindere rupes, pars mari, pars terra vehere intendebant, partesque diverse diversis aliis operibus indulgebant, cum celitus tanta confusione percussi sunt, ut qui omnes una eademque loquela deserviebant ad opus, ab opere, multis diversificati loquelis, desinerent, et nunquam ad idem commertium convenirent. Solis etenim in uno convenientibus actu eadem loquela remansit: puta cunctis architectoribus una, cunctis saxa volventibus una, cunctis ea parantibus una; et sic de singulis operantibus accidit. Quot quot autem exercitii varietates tendebant ad opus, tot tot ydiomatibus tunc genus humanum disiungitur; et quanto excellentius exercebant, tanto rudius nunc barbariusque locuntur

[E certo quasi tutto il genere umano si era mobilitato per l'iniqua impresa: chi dirigeva, chi progettava, chi tirava su i muri, chi li controllava con la livella, chi li intonacava con la cazzuola, chi badava a spaccar pietre, chi a trasportarle per mare e chi per terra, e altri si dedicavano a diverse altre operazioni; quando dal cielo furono colpiti da una tale confusione che, mentre prima lavoravano all'opera servendosi tutti di una sola e medesima lingua, ora, diversificati in tante lingue, dall'opera dovettero desistere, e non poterono mai più cooperare tutti alla stessa impresa. Infatti solo a quelli che lavoravano alla stessa operazione rimase una stessa lingua: per esempio una a tutti gli architetti, una a tutti quelli che rotolavano sassi, una a tutti quelli che li preparavano; e così accadde per quanti lavoravano alle singole operazioni. E quante erano le varie attività che concorrevano all'opera, in altrettanti idiomi in quel momento il genere umano si divide; e quanto più qualificata era l'attività, tanto più rozza e barbara è la lingua che ora parlano].

G.E. Onde dize Moysen en el onzeno capitulo queles partio Dios dauel logar por todas las tierras desta guisa: que quando ell uno demandaua ladriellos ell otro le daua bitumen, et quando ell otro pidie bitumen ell otrol aduzie agua, et quando ell otro dizie agua estel traye alguna delas ferramientas que y tenien o alguna otra cosa, de aguisa que nunca ell uno daua lo que ell otrol pidie, et quedaron de fazer la cibdad e la torre. Et por que fue alli mezclado e confondudo en muchos ell un lenguage que antes era de toda la tierra uno.

S.H. Dominus autem linguas eorum confudit, ut unusquisque vocem alterius non intelligeret sed aquam petenti, lapides, vel aliud quicquam porrigeret, sicque cessantes a proposito per orbem tripartitum in diuersis regionibus secundum linguarum suarum varietates disseminati sunt.

Mentre per Alfonso X e Vincenzo di Beauvais il linguaggio ha una finalità innanzitutto denotativa, che consiste nella rappresentazione verbale delle cose, per Dante essa ne ha una innanzitutto comunicativa, che consiste nella manifestazione reciproca dei concetti. Per cui la confusione divina non alte- 
ra il segno linguistico molecolarmente inteso, rompendo il rapporto semantico del suo lessico con gli oggetti (rapporto che, come abbiamo visto in Tommaso, rappresenta una funzione linguistica prerazionale e preumana) ma il sistema linguistico nel suo insieme, che si moltiplica in funzione dei diversi gruppi di artigiani, all'interno di ciascuno dei quali si continua invece a comunicare. Qui, però, all'interno di ciascun gruppo, la comunicazione è inutile poiché in essa non si riflette la natura economico-politica dell'essere umano, che si manifesta compiutamente solo attraverso la divisione del lavoro, e cioè attraverso la interazione comunicativa fra gruppi professionali diversi. È evidente, in questa rappresentazione sostanzialmente corporativa della comunicazione linguistica, la denuncia del prevalere degli interessi di parte (economica e/o politica) che ha distrutto (nella prospettiva di Dante) la solidarietà civile del comune. Come vuole Tommaso, «illi, qui sunt diversarum linguarum, non possunt bene convivere ad invicem», ed è appunto questo che accade fra i costruttori di Babele e, allegoricamente, fra i cittadini del popolo di Firenze: ogni gruppo di artigiani ha una sua lingua, rispetto alla quale quelle di tutti gli altri sono lingue straniere. Ciò che è straordinario è che Dante superi con una sola mossa intellettuale sia la concezione tradizionalmente lessicale del significato (per la quale «Nomina sunt consequentia rerum»), ${ }^{34}$ sia la antica dottrina politica dello «status in ordine triplex", che non contempla la divisione sociale del lavoro e i conflitti civili che da essa sono generati, conflitti divenuti ormai strutturali che rendono necessaria da una parte una sovranità di tipo globale (cioè prima il Regno poi l'Impero), capace di moderare il cieco e rapace egoismo dei gruppi economico-professionali e delle loro rappresentanze politiche, e dall'altra una lingua diastraticamente e diatopicamente unitaria (il «volgare illustre») che garantisca il flusso comunicativo all'interno della civitas, che è il fondamento naturale di qualunque solidarietà sociale. Dal Convivio al De Vulgari Dante matura una prospettiva teorica nella quale la società civile basata sulla

34. Vita Nuova XIII 4. Si vedano, sulla formula, le osservazioni di Pier Vincenzo Mengaldo (v. Lingua. Le teorie dantesche sulla lingua, Enciclopedia Dantesca, 1965). Nelle allusioni della Vita Nuova ai nomi di Beatrice (cap. II) e Giovanna (cap. XXIV) traspare una concezione ancora sostanzialmente lessicale del significato del nome (come l'autore dichiara, citando Aristotele, in Conv. III xi 1: «la diffinizione è quella ragione che 'l nome significa»). In particolare in riferimento alla seconda, si parla di una imposizione del nome (XXIV 3: «lo nome di questa donna era Giovanna, salvo che per la sua bieltade imposto l'era nome Primavera; e così era chiamata ... Io [Amore] mossi lo imponitore del nome a chiamarla così) che potrebbe essere riecheggiamento di suggestioni modiste: «L'imposizione originaria delle espressioni linguistiche costituisce il momento fondatore: pur non essendo un tratto originale della loro teoria, la codificazione originaria acquista una modulazione particolare con i Modisti» (Costantino Marmo, op. cit., p. 328). La disinvoltura con cui i nomi vengono imposti, nella Vita Nuova, alle signore amate dai poeti fa poi pensare ad un atteggiamento sostanzialmente parodico, sia nei confronti della tradizione grammaticale che nei confronti della tradizione scritturale (nella quale la «impositio nominum» ha carattere liturgico e sacrale). 
divisione del lavoro, lo stato moderno ${ }^{35}$ e la lingua nazionale appaiono indissolubilmente vincolati e connessi l'uno all'altro.

\section{Il superamento del dualismo diglossico: la Commedia}

Uno degli aspetti più discussi e discutibili del De Vulgari è l'aspra condanna dei volgari municipali, cioè delle lingue materne, che gli scrittori italiani dovrebbero abbandonare per adottare lo stile più elevato. Si tratta di un tema svolto esclusivamente nel trattato latino, e che produce una ulteriore dicotomia rispetto al Convivio. ${ }^{36}$

Mentre nel Convivio l'amore nei confronti del volgare proprio è necessaria condizione morale per la sua difesa nei confronti del latino, nel De Vulgari il disprezzo nei confronti del volgare proprio è condizione di una razionale considerazione del linguaggio, e più avanti sarà, soprattutto, necessaria condizione morale per la elaborazione del «volgare illustre». Indipendentemente dalla precoce intuizione di una situazione linguistica caratterizzata dalla opposizione lingua-dialetti, come effettivamente sarà quella italiana, ciò che colpisce di questa antitesi è che nel Convivio la nozione di lingua italiana appare molto meno conflittuale, al suo interno, di quella che invece domina nel De Vulgari. L'unica diglossia cui si allude è, come nella Vita Nuova, quella fra volgare e latino. Si prenda il famoso brano in cui viene evocata la situazione dell'esule ramingo fra le diverse zone della penisola Conv. I in 4:

35. Intendo per «stato moderno" uno stato definito a partire dalla identità nazionale, il territorio e la lingua, gli elementi che Dante prende in considerazione nel De Vulgari nella sua ricerca del «volgare illustre». Sull'idea di comunione linguistica come fondamento della nazione italiana, si veda Antonino Pagliaro, Ulisse. Ricerche semantiche sulla 'Divina Commedia', Messina-Firenze: D’Anna, 1967, p. 530-532.

36. Si confrontino questi due brani:

Conv. I XII 1-2. Se manifestamente per le finestre d'una casa uscisse fiamma di fuoco, e alcuno dimandasse se là dentro fosse il fuoco, e un altro rispondesse a lui di sì, non saprei bene giudicare qual di costoro fosse da schernire di più. E non altrimenti sarebbe fatta la dimanda e la risposta di colui e di me, che mi domandasse se amore a la mia loquela propria è in me e io li rispondesse di sì, apresso le su proposte ragioni. Ma tuttavia, e a mostrare che non solamente amore ma perfettissimo amore di quella è in me, ed a biasimare ancora li suoi aversarii ciò mostrando a chi bene intenderà, dirò come a lei fui fatto amico e poi come l' amistà è confermata.

De Vulgari I vi 2. In hoc, sicut etiam in multis aliis, Petramala civitas amplissima est, et patria maiori parti filiorum Adam. Nam, quicunque tam obscene rationis est ut locum sue nationis delitiosissimum credat esse sub sole, hic etiam pre cunctis proprium vulgare licetur, idest maternam locutionem, et per consequens credit ipsum fuisse illud quod fuit Ade. [Per questo aspetto, come anche per tanti altri, Pietramala è una città grandissima, e patria della maggior parte dei figli di Adamo. Infatti, chiunque ha una mente così oscena da credere che il posto dov'è nato sia il più delizioso sulla terra, costui pregia anche il proprio volgare, cioè il parlar materno, al di sopra di ogni altro, e di conseguenza crede che sia stato proprio quello che fu di Adamo]. 
Poi che fu piacere de li cittadini de la bellissima e famosissima figlia di Roma, Fiorenza, di gittarmi fuori del suo dolce seno - nel quale nato e nutrito fui in fino al colmo de la vita mia, e nel quale, con buona pace di quella, desidero con tutto lo cuore di riposare l'animo stancato e terminare lo tempo che m'è dato - , per le parti quasi tutte a le quali questa lingua si stende, peregrino, quasi mendicando, sono andato, mostrando contra mia voglia la piaga de la fortuna, che suole ingiustamente al piagato molte volte essere imputata.

Notevolissima è qui l'idea di una lingua, quella in cui sta scrivendo, che afferisce ad un territorio sul quale essa si proietta in un nesso organico fra lingua letteraria (potenzialmente di una corte unitaria, cioè uno stato e quindi una cultura nazionali, dirà il De Vulgari) ${ }^{37}$ e geografia. Per intendere questa estensione alla intera penisola del toscano in cui sta scrivendo, bisogna tener conto di due fenomeni che condizionano la percezione di Dante: il primo è la toscanizzazione dei testi siciliani, per cui egli leggeva i poeti di Sicilia non nel siciliano originario in cui furono scritti, ma nel rimaneggiamento toscano cui i testi furono sottoposti nel corso della loro trasmissione; il secondo è l'adozione della lingua letteraria risultante da tale processo da parte dei lirici bolognesi, e in particolare di Guinizzelli. La lingua in cui sta scrivendo gli appare, per ciò, non esclusiva di Firenze o della Toscana, ma estesa alla intera penisola. Ad essa attingono da sud a nord poeti che condividono non solo la lingua letteraria ma anche l'ideologia ad essa sottesa, in cui l'amore è, come abbiamo visto nella Vita Nuova, fonte di vita morale e di ispirazione poetica. Ciò che conta, però, è il fatto che questa lingua, per il fatto di essere panitaliana, non è, per questo, meno «congiunta con le più prossime persone, sì come con li parenti e con li propri cittadini e con la propria gente» (Convivio I XII 5). Essa sembra diffondersi in modo uniforme sia sull'asse diastratico che su quello diatopico. Per il fatto di essere, o aspirare ad essere, lingua nazionale e di stato, non cessa di essere lingua materna. Quando gli scrittori italiani del 400, da Lorenzo de' Medici a Masuccio Salernitano a Pulci, a Giovanni degli Arienti parlano di lingua materna, alludendo alla lingua in cui scrivono, aderiscono al modello teorico del Convivio, per il quale il concetto di lingua propria e materna include il livello alto e comune, cioè quello dell'italiano inteso come lingua letteraria. ${ }^{38} \mathrm{Nel}$ De Vulgari tale modello viene, invece,

37. Il significato politico-culturale del De Vulgari è stato perfettamente indicato da Karl Otto Apel, L'idea di lingua nella tradizione dell'umanesimo da Dante a Vico, Bologna: Il Mulino, 1975, p. 157: «Il peculiare significato del trattato dantesco De vulgari eloquentia risiede nel fatto che, per quanto infinitamente complicato, il processo consistente nel giustificare in poesia l'esistenza d'una lingua nazionale viene posto contemporaneamente nella luce d'una consapevole programmatica. Con tale consapevole formazione ideale, che si ricollega alle tecniche classiche del discorso, Dante ha prospettato il problema culturale caratteristico dell'epoca a lui successiva: la trasformazione dell'unitaria civiltà latina dell'Occidente medievale nel sistema letterario delle lingua nazionali della nuova Europa».

38. Rinvio sul tema al mio Raffaele Pinto, La donna come alterità linguistica in Sergio ZatTi (a cura di), La rappresentazione dell'altro nei testi del Rinascimento, Lucca: Pacini-Fazzi, 1998, p. 13-32. 
ribaltato, e la nozione di lingua propria e materna si identifica con il polo basso e municipale, cioè con la lingua parlata. È interessante osservare come la scoperta del De Vulgari, nel 500, produca, alla fine del dibattito teorico, uno spostamento di significato del concetto di lingua materna, che non è più la lingua letteraria comune, come per gli scrittori quattrocenteschi, e diventa senz'altro il dialetto locale, d'accordo con il modello diglossico del trattato latino. ${ }^{39} \mathrm{Al}$ tendenziale monolinguismo volgare del Convivio si oppone dunque la tendenziale diglossia volgare del De Vulgari, che implica però una distanza molto minore fra i due poli estremi di quella implicita nella diglossia latino-volgare.

Un accenno al superamento della diglossia, infatti, credo che sia riscontrabile anche nel trattato latino, quando del poeta lombardo Sordello da Goito, italiano che scrive in provenzale, si dice che

I xv 2. Tantus eloquentie vir existens, non solum in poetando sed quomodocumque loquendo patrium vulgare deseruit

[il quale, da quell'uomo di alta eloquenza che era, abbandonò il volgare della sua patria non solo in poesia ma in qualunque forma di espressione].

Il senso del passo è controverso. È chiaro, però, che a proposito di Sordello viene postulata, come un merito conseguente alla sua alta eloquenza, l'identità di linguaggio, sia nella scrittura poetica che nella oralità. Pur trattandosi di un ulteriore argomento contro il volgare municipale (che Sordello abbandona in tutti i registri espressivi), il poeta mantovano rappresenterebbe un esempio soggettivo di realizzato monolinguismo, grazie al quale l'antagonismo scrittoparlato viene neutralizzato in virtù di un perfetto dominio dell'eloquenza in tutti i suoi registri. L'incontro di Sordello con Virgilio, nel Purgatorio, nel nome di una comune identità sia geografica che linguistica, sembra finalizzato appunto a neutralizzare ogni indebita sopravvalutazione delle differenze di lingua, senżaltro irrilevanti rispetto ad una sostanziale italianità di cultura linguistica. ${ }^{40}$

39. Si veda, per esempio, Tasso: «Perché a le piagge ove ’l Sebeto inonda /vicin son nato, ove il pianeta eterno / non distempera mai la state e 'l verno, / né gli odorati rami il gelo sfronda, / pregio il tosco sermon che tanto abbonda / di colti detti; co 'l parlar materno /l'altre favelle ho parimenti a scherno / d'udir fra l'alpe e 'l mar che ne circonda” (Rime, 828, 1-8).

40. La concezione "trascendentale" dell' italiano (inteso come repertorio di registri al servizio di un soggetto poetico espressivamente sovrano) viene implicitamente insinuata in De Vulgari II I-II. Se ne è accorto P.V. Mengaldo, che nella "Introduzione al "De Vulgari Eloquentia"», p. 90-91 (in Linguistica e retorica in Dante, Pisa: Nistri-Lischi, 1978, p. 11-123) osserva: «Si legga tra le righe di II I e II... Se ne deduce che il poeta capace di stile sommo ha però libertà di scelta anche tra gli stili minori, parimenti giustificati nel loro ambito, purché naturalmente adegui stile a materia e volgare a stile; e che insomma uno stesso individuo può poetare alternamente, quando stia alle regole del gioco, in stile sublime, medio e basso e dunque in volgare illustre, mediocre e umile». Stranamente, però, questa apertura teorica allo sperimentalismo espressivo della Commedia viene considerata dallo studioso come un limite del trattato e uno dei motivi della sua incompiutezza: «non si capisce ... in che modo un individuo costituzionalmente dignissimus possa concepire conceptiones men che ottime, possa cessare pro tempore di essere tale, cioè di essere se stesso, per cogliere i fiori degli stili 
Ma il superamento del paradigma diglossico si produce compiutamente solo nella Commedia, cioè in un testo che, presentandosi come una enciclopedia di stili e, in qualche misura, anche di lingue, è esempio in atto della transitabilità soggettiva di lingue e registri, che nel loro insieme rappresentano il repertorio dell'io, che liberamente seleziona, di volta in volta, quella o quello più adeguati alle proprie esigenze espressive. L'italianità trascendentale del Poema, proclamata fin dal principio ("di quella umile Italia fia salute...»), ${ }^{41}$ è soggiacente alle pur così varie modalità espressive in esso operanti, di cui ciò che soprattutto importa è la soggettiva possibilità di attraversamento. La fiorentinità del Poema è, in tale prospettiva, elemento puramente accidentale; elemento sostanziale è invece che, a partire da un volgare proprio determinato, tutti i registri culturalmente possibili vengano attraversati dal soggetto che si esprime. Più in generale, si può dire che nella Commedia Dante neutralizza tutte le dicotomie che, relativamente al linguaggio, hanno articolato il suo pensiero. Più che di monolinguismo, converrà parlare, perciò, di radicale relativismo, per il quale il linguaggio cessa di essere terreno di conflitto, mentale o politico. Un esempio clamoroso di relativismo, cioè di attraversamento soggettivo di lingue e registri è quello di Virgilio che, pur essendo rappresentante sommo della grammatica latina, non esita a rivolgersi in dialetto lombardo ad Ulisse. Un altro esempio ce l'abbiamo nel personaggio di Brunetto Latini, nel quale riaffiora la polemica contro $\mathrm{i}$ «malvagi uomini d'Italia» che disprezzano il volgare proprio, se, come credo con Pézard, ${ }^{42}$ la sua sodomia è manifestazione di un atteggiamento contronaturale anche sul piano del linguaggio. Bisogna però osservare che Brunetto, autore di testi in volgare toscano oltre che in francese, era stato coinvolto nella polemica del De Vulgari contro il municipalismo poetico toscano:

I XIII 1. Brunectum Florentinum, quorum dicta, si rimari vacaverit, non curialia sed municipalia tantum invenientur

[Brunetto Latini i cui versi, se ci sarà spazio per frugarci dentro, si riveleranno non cuariali ma solo municipali],

per cui grava su di lui una doppia condanna: relativamente al volgare proprio, per aver usato un volgare altrui, e relativamente al volgare illustre, per aver usato un volgare municipale. Ebbene l'episodio dell'Inferno, pur nel quadro della condanna morale della sodomia, ne riscatta l'immagine e la memoria su entrambi i piani, come intellettuale fiorentino, per aver insegnato a Dante, col suo magistero, "come l'uom s'etterna», e come scrittore di un opera in lingua

inferiori... E forse non si capisce neppure come possa e voglia, lui che è italiano a pieno titolo, farsi o rifarsi uomo della regione e del municipio».

41. Mi inclinerei, quindi, per una lettura autoreferenziale del veltro, in Inf. I: come già nel pastore di Conv., I XI, Dante si raffigura come novello redentore della nazione italiana, attraverso la lingua e la poesia. Sulla autoreferenzialità del veltro si veda soprattutto Paolo Baldan, «Per un veltro dal substrato materico: Inferno, I», Italianistica, XXI, n. 2-3, 1992, p. 297-314.

42. André Pezard, Dante sous la pluie de feu, Paris: Vrin, 1950. 
francese: «il mio Tesoro». La palinodia, rispetto alle condanne dei due trattati, non potrebbe essere più clamorosa!

E clamorosa è anche la ritrattazione, rispetto alla lingua materna, che leggiamo nell'episodio di Arnaut Daniel (Purg. XXVI), che deve il suo primato al fatto di essere «il miglior fabbro del parlar materno», espressione nella quale la lingua propria e materna viene riabilitata contro la condanna che abbiamo appena visto del De Vulgari. L'immagine del fabbro, infatti, ricorda da vicino quella che Dante utilizza, in Conv. I XIII 4, per dimostrare che il volgare proprio fu «cagione del suo essere»:

Questo mio volgare fu congiugnitore de li miei generanti, che con esso parlavano, sì come 'l fuoco è disponitore del ferro al fabbro che fa lo coltello; per che manifesto è lui essere concorso a la mia generazione, e così essere alcuna cagione del mio essere.

Ma non è solo una rivalutazione della lingua materna. Il fatto che Guinizzelli e Arnaut si presentino assieme, nella cornice dei lussuriosi, ustionati da un incendio come i sodomiti nell'Inferno dalla pioggia di fuoco, evoca una ideale genealogia della lirica in volgare di cui Dante si sente erede ed elimina qualunque sospetto di antagonismo fra lingua propria e lingua altrui, almeno nell'ambito del dominio romanzo che già nella Vita Nuova appariva come una unica tradizione lirica.

Ed arriviamo finalmente alle terzine pronunciate da Adamo in Par. XXVI, che rappresentano il punto conclusivo della riflessione linguistica di Dante e nelle quali la neutralizzazione dei dualismi teorici e diglossici viene solennemente proclamata:

Tu vuogli udir... / l'idioma ch'usai e che fei...

La lingua ch'io parlai fu tutta spenta

innanzi che a l'ovra inconsummabile

fosse la gente di Nembròt attenta:

ché nullo effetto mai razionabile,

per lo piacere uman che rinovella

seguendo il cielo, sempre fu durabile.

Opera naturale è ch'uom favella;

ma così o così, natura lascia

poi fare a voi secondo che v'abbella.

Pria ch'i' scendessi a l'infernale ambascia,

I s'appellava in terra il sommo bene

onde vien la letizia che mi fascia;

e El si chiamò poi: e ciò convene,

ché l'uso dei mortali è come fronda

in ramo, che sen va, e altra vene.

Il passo ha attirato l'attenzione degli studiosi soprattutto per la palinodia relativa al mito dell'ebreo come lingua sacra ed immutata, ed al mito di Babele come origine della proliferazione delle lingue, entrambi sacrificati all'idea 
della integrale storicità del linguaggio umano, che fin dal principio è iscritto sotto il segno del mutamento. Nel quadro della presente riflessione, però, sono altri i punti prevalenti di interesse, e cioè quelli che alludono ad una riduzione dei dualismi e ad una concezione del linguaggio che ne fa un prodotto e un effetto della libertà umana. Si osservi innanzitutto che Adamo parla del linguaggio che usò e che fece. Le due espressioni rinviano ai due concetti antagonistici dell' uso e dell'arte, cioè del volgare e della grammatica, qui finalmente unificati nella prospettiva di un soggetto che usa e fa la lingua, e che quindi detiene sia le risorse della espressione (la parole, diremmo saussurianamente) sia le regole della grammatica (ossia la langue). Adamo non ha ricevuto la lingua né da una nutrice né da Dio, l'ha semplicemente prodotta, così come produce, in quanto umano, gli strumenti che sono necessari alla sua esistenza. Fabbro, quindi, come Arnaut Daniel, ma in un senso più radicale, perché la lingua che lui fabbrica non gli preesiste come volgare proprio e materno, ma bensì la crea, si direbbe, dal nulla. ${ }^{43}$ Ecco allora il colpo di scena che abbatte con una audace mossa teorica tutti i dualismi faticosamente affrontati nelle opere precedenti: la natura è responsabile non delle lingue (in qualunque senso considerate, gli idiomi e i volgari del De Vulgari) ma semplicemente della facoltà di linguaggio, cioè la locutio del De Vulgari, la capacità articolatoria che fa dell'essere umano un soggetto parlante. Il fatto che parli «così o così», cioè in questa lingua o quell'altra, in un registro elevato e formale oppure in uno spontaneo e informale, tutto ciò dipende interamente dalle scelte dei parlanti, che cambiano lingua e registri in funzione di scelte radicalmente libere (o comunque storicamente motivate). Tale concetto della arbitrarietà linguistica si iscrive certo nell'orizzonte teorico del convenzionalismo aristotelico, ed anzi la fonte più diretta di Dante sembra, ancora una volta, il commento di Tommaso al Perihermeneias, in cui leggiamo:

De interpretatione, lib. 11.6 n. 8. Aristoteles dicit quod omnis oratio est significativa, non sicut instrumentum virtutis, scilicet naturalis: quia instrumenta naturalia virtutis interpretativae sunt guttur et pulmo, quibus formatur vox, et lingua et dentes et labia, quibus litterati ac articulati soni distinguuntur; oratio autem et partes eius sunt sicut effectus virtutis interpretativae per instrumenta praedicta. Sicut enim virtus motiva utitur naturalibus instrumentis, sicut brachiis et manibus ad faciendum opera artificialia, ita virtus interpretativa utitur gutture et aliis instrumentis naturalibus ad faciendum orationem. Unde oratio et partes eius non sunt res naturales, sed quidam artificiales effectus.

Posto che le lingue concretamente parlate e scritte, in tutti i loro aspetti semiotici (la «virtus interpretativa»), sono solo «effetto razionabile» («artificia-

43. Anche nel De Vulgari Adamo fabbrica la lingua: «Fuit ergo hebraicum ydioma illud quod primi loquentis labia fabricarunt», ma non nel senso integrale che ha qui il verbo "fare», «l'idioma ... ch'io fei», giacché con la sua anima Dio aveva creato anche "certam formam locutionis». Nel Paradiso, invece, Dio non ha alcun bisogno di creare un lingua poiché ha provvisto Adamo degli strumenti naturali per farlo da sé. 
les effectus»), l'elemento naturale del linguaggio è rappresentato puramente dagli strumenti anatomici necessari per produrre il suono ed articolare la voce (guttur et pulmo ... lingua et dentes et labia) : «opera naturale è», semplicemente, "ch'uom favelli», ma che tale possibità fisiologica produca una lingua oppure un'altra, dipende dalla arbitrarietà delle scelte umane (variabili in funzione delle concrete situazioni storico-sociali). Come si vede, la dissacrazione di ogni mistica della lingua come spirito o genio di un popolo che ne predetermina il destino storico è totale. Ma, pur nel solco della teoria aristotelico-tomista del linguaggio, Dante innova e ridefinisce potentemente i concetti. Infatti la libertà espressiva che Adamo rivendica, per sé e per l'umanità, è una libertà innanzitutto estetica, quindi relativamente poco soggetta perfino alle determinazioni sociali o politiche, che nei due trattati apparivano così decisive. Ce ne accorgiamo attraverso il verbo che Dante utilizza per indicare questa radicale libertà dei parlanti: «secondo che v'abbella», cioè non secondo i bisogni e le necessità, ma secondo il puro principio del piacere, quello che si esalta al massimo grado nell'esercizio della poesia, che di nuovo appare come la principale delle istituzioni del linguaggio umano, ma non più in quanto finalizzata a cementare, attraverso il "volgare proprio», la società civile, come nel Convivio, né in quanto finalizzata alla formazione dello stato nazionale, come nel De Vulgari, ma in quanto dimensione primordiale della libertà del soggetto, un soggetto che, almeno utopicamente, usa e $f a$, come Adamo, la propria lingua. Indubbiamente, il plurale che usa il personaggio, «vi abbella», allude a comunità di linguaggio, non a soggetti isolati. Il verbo abbella però aggiunge alla dimensione sociale del linguaggio suggestioni individuali e personali che sarebbe grave errore non cogliere. Abbella è provenzalismo che Dante aveva usato nella parlata, in provenzale, di Arnaut Daniel, in Purg. XXVI, in una espressione, Tant miabellis vostre cortes deman, che è citazione da un incipit di Folchetto da Marsiglia. La memoria lirica e trovadorica con cui la riflessione sul linguaggio era iniziata nella Vita Nuova, riaffiora qui nella evocazione del primo parlante e dell'idioma "quo vir sine matre, vir sine lacte ... creditur usus». Notevolissimo e il fatto che tale «vir sine matre» parli come «il miglior fabbro del parlar materno». Ed altrettanto notevole è che il nome di Dio, il primo nome pronunciato da Adamo, secondo il De Vulgari (I IV 4), sia soggetto anch'esso, come ogni altro nome, all'arbitrio del piacere umano, cioè al mutare dei costumi e dei linguaggi. Si confronti la teorizzazione relativa alla imposizione dei nomi, nella Vita Nuova, per la quale i nomi sono vincolati alle persone perché ne contengono, in qualche misura, i valori caratterizzanti, con l'assoluta indipendenza, qui, del nome di Dio (nientedimeno!) dal suo referente.

Al termine di un periplo in cui ha attraversato tutti i saperi alla ricerca delle origini e delle funzioni del linguaggio, Dante scopre ciò che fin dal principio aveva intuito, e cioè che al di là delle dicotomie, che irrazionalmente frantumano in istanze contrapposte il linguaggio umano, il territorio in cui esso si rivela come l'autentico ed esclusivo territorio della libertà del soggetto, è l'esercizio della poesia. 